This is a post-peer-review, pre-copyedit version of an article published in Microchimica Acta. The final authenticated version is available online at:

https://dx.doi.org/10.1007/s00604-019-3323-y 


\title{
A Nitrocellulose Paper Strip for Fluorometric Determination of Bisphenol A Using Molecularly Imprinted
} Nanoparticles

\section{Recep Üzek $^{\mathrm{a}, \mathrm{b}}$, Esma Sari ${ }^{\mathrm{a}, \mathrm{b}}$, Serap Şenel ${ }^{\mathrm{b}}$, Adil Denizli ${ }^{\mathrm{b}}$, Arben Merkoçi*,a,c}

${ }^{\text {a }}$ Catalan Institute of Nanoscience and Nanotechnology (ICN2), CSIC and BIST, Campus UAB, Bellaterra,

$$
\text { Barcelona 08193, Spain }
$$

${ }^{\mathrm{b}}$ Hacettepe University, Faculty of Science, Department of Chemistry, 06800, Ankara, Turkey

${ }^{\mathrm{c}}$ ICREA, Pg. Lluís Companys 23, 08010 Barcelona, Spain

\begin{abstract}
The authors describe a test stripe for fluorometric determination of the endocrine disruptor bisphenol A (BPA). Graphene quantum dots (GQDs) were immobilized on molecularly imprinted nanoparticles which then were placed on nitrocellulose paper. The GQDs display blue fluorescence (with excitation/emission peaks at 350/440 $\mathrm{nm}$ ) which is reduced in the presence of BPA. The test stripe has a $43.9 \pm 0.8 \mu \mathrm{g} \cdot \mathrm{L}^{-1}$ limit of detection in case of water samples. The stripe was applied to the determination of BPA in (spiked) tap water and sea water, and the LODs were found to be $1.8 \pm 0.2 \mu \mathrm{g} \cdot \mathrm{L}^{-1}$ and $4.2 \pm 0.5 \mu \mathrm{g} \cdot \mathrm{L}^{-1}$, respectively. Structural analogs of BPA, such as aminophenol, phenol, hydroquinone and naphthol were found not to interfere.
\end{abstract}

Keywords: Graphene quantum dot, endocrine disruptor, fluorescence quenching, paper sensor, molecular recognition 


\section{Introduction}

Bisphenol A, (BPA, 4,4'-(propane-2,2-divinyl)diphenol) formed by the condensation reaction between one acetone and two phenol groups, is an indispensable organic chemical (monomer) in the production of polycarbonate, polystyrene resins, epoxy resins, plastics, polysulfones, and polyesters used as plasticizers, fire retardants and coating agents [1]. BPA have been widely utilized in various products such as water bottles, containers, eyeglass lenses, automotive parts, CDs, beverage cans, tableware, microwave ovenware, dental sealants, packing materials, baby bottles and paper [2,3]. BPA is among the most important chemicals globally, with over six billion pounds produced each year [4] because of its pivotal position in household and industry products [5]. In contrast to the multiple applications contemplated for BPA, there is a growing concern about the usage of BPA because it can mimic the properties of estrogens, endogenous hormones, and androgens by binding to estrogen receptors [6,7]. Therefore, BPA is denoted as an endocrine-disrupting compound [7]. BPA can be hydrolyzed by exposing the ester bonds of the materials with heat, acid, or base, and thus releasing BPA in the environment. Even the low levels of BPA may reportedly cause several adverse health effects such as cardiovascular diseases, obesity, cancer, neurotoxicity, and reproductive disorders in adults [8,9]. Therefore, the detection of trace amounts of BPA is essential; a number of techniques have been employed, such as high performance liquid chromatography (HPLC) [10], enzyme-linked immunosorbent assay (ELISA) [11,12], Surface-enhanced Raman spectroscopy (SERS) [13], electrochemical sensors [14,15], gas chromatography-mass spectrometry (GC/MS) [16], gas chromatographytandem mass spectrometry (GC/MS/MS) [17], liquid chromatography-mass spectrometry (LC/MS) [18]. However, these techniques require sophisticated and expensive instruments used by professionals, and the operation is complex and time consuming. Hence, a simple and effective method to detect BPA in environmental samples is highly desirable.

Graphene and its derivatives have attracted considerable interest because of their extraordinary optical and electronic properties and biocompatibility [19]. Nanometer-sized graphite derivatives called graphene quantum dots (GQDs) have been the subject of intensive research because of their remarkable physical, mechanical, and optoelectronical properties [20,21]. Compared to semiconductor quantum dots, GQDs are advantageous because they exhibit stable photoluminescence, low cytotoxicity, and excellent biocompatibility [22,23]. In addition, GQDs carry massless charge, and exhibit profound edge effects, chemically inertness, and reduced photobleaching [24]. Due to improved physical, mechanical, and optoelectronic properties, GQDs exhibit better performance in application as compared to conventional semiconductor quantum in addition to being environment friendly. Moreover, GQDs have been used in a wide variety of applications such as in photocatalytic devices [25,26], drug delivery [27], biosensing [28] and cellular imaging [29,30].

Molecular imprinting is an artificial (non-biological) molecular recognition approach based on the "molecular key and lock" principle. One of the most attractive advantages of molecular imprinting is its adoptability to a wide range of polymers such as cryogels [31], monoliths [32], microparticles [33], and nanoparticles [34] etc. owing to their desired selectivity, physical robustness, and thermal stability, as well as low cost and easy preparation. Molecularly imprinted polymers (MIPs) have been extensively utilized in sensor systems; however, imprinted nanoparticles were chosen because of their inherent advantages of sensitivity, selectivity, low cost, and easy operation [35]. 
Herein, we developed a fluorescent-based nanosensor for the detection of BPA (Fig. 1). BPA imprinted nanoparticles which have specific recognition cavities for BPA were synthesized by a mini-emulsion polymerization method. GQDs were used as a fluorescent agent and synthesized by hydrothermal pyrolysis. Coupling using N-(3-Dimethylaminopropyl)-N'-ethylcarbodiimide hydrochloride (EDC) and N-Hydroxysuccinimide (NHS) was applied for the immobilization of GQDs onto the NPs. After characterization of the NPs, BPA detection was performed in an aqueous solution or on a nitrocellulose paper. The selectivity and specificity of the sensor system for BPA detection in tap water and seawater were investigated by spiking BPA as well as its structural analogs, such as aminophenol, phenol, hydroquinone and naphthol into tap water or seawater.
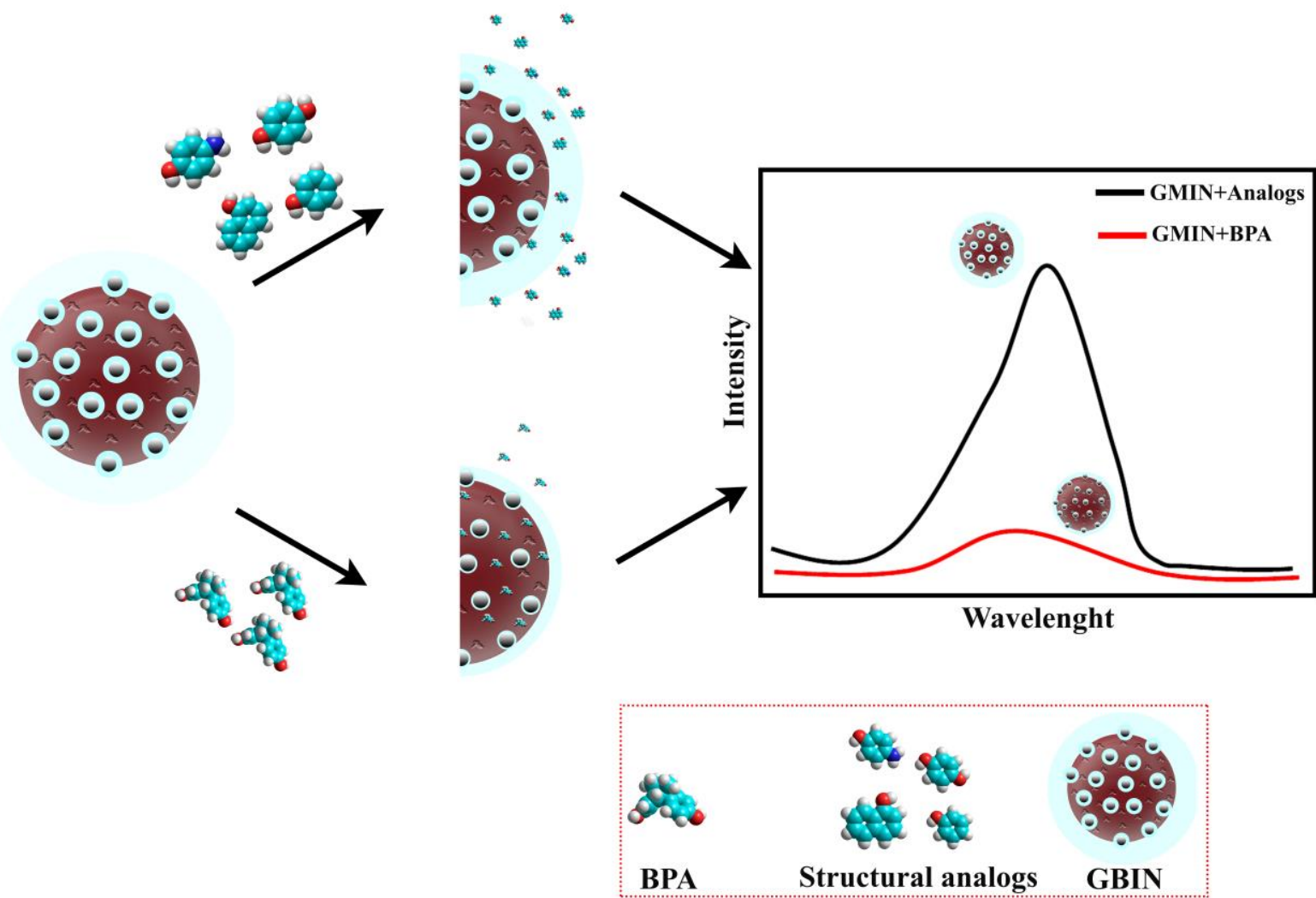

Fig. 1. BPA sensing mechanism based on the fluorescence quenching by the binding of targets in specific recognition sites.

\section{Materials and methods}

\section{Chemicals and apparatus}

BPA, phenol, naphthol, aminophenol, hydroxyquinone, L-glutamic acid (Glu), NHS, quinine sulfate, EDC, Laspartic acid (Asp), vinyl imidazole (VIM), ethylene glycol dimethacrylate (EDMA), ammonium persulfate (APS), sodium bisulfite, sodium dodecyl sulfate (SDS), and polyvinyl alcohol (PVA, MW 30.000-70000), were purchased from Sigma (Madrid, Spain, http://www.sigmaaldrich.com). All chemicals were of analytical grade and used as received unless otherwise noted. The other reagents were analytical grade, and were from Sigma (Madrid, Spain). Milli-Q water for aqueous solutions was obtained from a Milli-Q system (>18.2 $\mathrm{MW} \mathrm{cm}^{-1}$ ) purchased from Millipore (https://www.merckmillipore.com).

The solutions were homogenized by a Qsonica Q125 Sonicator (Qsonica, Newtown, USA, https://www.sonicator.com). The morphology, size and distribution of nanoparticles were determined by Nano Zetasizer (NanoS, Malvern Instruments, London, UK, https://www.malvernpanalytical.com) and scanning 
transmission electron microscopy (S-TEM, FEI Tecnai F20, FELMI-ZFE, Graz, Austria, https://www.felmizfe.at).

The chemical structure of nanoparticles was characterized via Fourier transform infrared spectrometry (Bruker PMA 50 with Tensor 27, Coventry, UK, https://www.bruker.com) and X-ray photoelectron spectroscopy (XPS; Phoibos-150 analyzer/spectrometer, SPECS GmbH, Berlin, Germany, http://www.specs.de).

A SpectraMax M2e spectrophotometer (Molecular Devices, Sunnyvale, CA, https://www.moleculardevices.com) was used for UV-VIS absorbance and photoluminescence measurements. The nanoparticles were precipitated by Sigma centrifuge (Sigma® 2-16P, Fisher Bioblock Scientific, DE, https://www.sigma-zentrifugen.de).

\section{Preparation of graphene quantum dots (GQDs)}

Glutamic acid and aspartic acid were used to synthesize graphene quantum dots (GQDs) via hydrothermal pyrolysis. Briefly, $2.0 \mathrm{~g}$ glutamic acid and $100 \mathrm{mg}$ aspartic acid in a $10 \mathrm{~mL}$ beaker were heated to $185^{\circ} \mathrm{C}$ by using a silicone oil bath. The solid mixture completely melted after $15 \mathrm{~min}$ and at liquid solution of GQDs was obtained clearly. The solution was added into Milli-Q water $(25 \mathrm{~mL})$. A standard quinine sulfate $(50 \mathrm{mM})$ solution $(\varphi=54 \%)$ was used to calculate the quantum yield $(\varphi)$ of GQDs.

\section{Preparation of BPA imprinted polymeric nanoparticles}

Mini-emulsion method was used for preparation of BPA imprinted nanoparticles (BIN). Firstly, a pre-complex was prepared after determination of the functional monomer to template ratio (Electronic Supplementary Material, Table S1 and Fig. S1), and then two aqueous solutions were prepared (Fig. S2). For the first aqueous phase, PVA $(94 \mathrm{mg})$, SDS (14 mg), and sodium bicarbonate $(12 \mathrm{mg})$ were dissolved in $5.0 \mathrm{~mL}$ Milli-Q water. The second aqueous phase was obtained by dissolving PVA (50 mg) and SDS (50 mg) in $100 \mathrm{~mL}$ Milli-Q water. BPA (34 mg, $0.15 \mathrm{mmol}$ ) was dissolved in functional monomer (VIM, $220 \mu \mathrm{L}, 2.4 \mathrm{mmol}$ ) to prepare the pre-complex. Acrylamide (AAm, $100 \mathrm{mg}, \sim 1.4 \mathrm{mmol}$ ) was then added as a co-monomer to obtain amine groups on the surface of polymeric nanoparticles; EDMA $(1.05 \mathrm{~mL})$ as a crosslinking monomer was added into the pre-complex solution to form the organic phase. The organic phase was mixed with the first aqueous phase and the mixture was sonicated in an ice bath for 20 min with $90 \%$ amplitude to obtain mini-emulsions. After sonication, the second aqueous phase was added rapidly to avoid degradation of the emulsions and stirred magnetically $(500 \mathrm{rpm})$ at $40{ }^{\circ} \mathrm{C}$. When the temperature of the polymerization mixture reached $40{ }^{\circ} \mathrm{C}$, the initiators sodium bisulfite (58 $\mathrm{mg}$ ) and ammonium persulfate $(63 \mathrm{mg})$ were added respectively and the polymerization process was allowed to proceed for $24 \mathrm{~h}$. In order to terminate the polymerization, coarse centrifugation was applied for $30 \mathrm{~min}$ at $5000 \mathrm{rpm}$ to remove the large sized particles in nanoparticle solution. Centrifugation was carried out at $15000 \mathrm{rpm}$ for one hour to sediment the nanoparticles and the excess surfactant and non-reacting monomers were removed in the supernatant. The precipitate obtained in each settling process was dissolved in a mixture of Milli-Q water: ethanol (1:1 v/v) with sonication and then centrifuged again to remove the template molecule (BPA), surfactants, nonreactive monomer residues and initiator pairs. This process was repeated six times and then the process of washing with Milli-Q water was performed three times. The concentration of nanoparticles was adjusted to $30 \mathrm{mg} \cdot \mathrm{mL}^{-1}$ by dispersing in Milli-Q water and the nanoparticles were stored at $4{ }^{\circ} \mathrm{C}$ until use. Non-imprinted nanoparticles (BNIN) were obtained following the same procedure without the addition of BPA in the pre-complex.

\section{Characterization of nanoparticles}

The structural characterization of nanoparticles was carried out by X-ray photoelectron spectroscopy (XPS), and Fourier transformed infrared spectroscopy (FTIR). The size distribution of nanoparticles was performed by Nano 
Zetasizer. The surface morphology of nanoparticles was characterized by a Tecnai G2 F20 transmission electron microscope (TEM; FEI) in normal mode and by scanning electron microscopy (SEM). Absorbance and emission spectra were recorded by UV-VIS spectrophotometry.

The quantum yield of GQDs was calculated according to the following equation, using quinine sulfate (QS) as a reference:

$$
\Phi_{x}=\Phi_{S T}\left(\frac{A_{S T}}{A_{X}}\right)\left(\frac{I_{X}}{I_{S T}}\right)\left(\frac{\eta_{X}^{2}}{\eta_{S T}^{2}}\right)
$$

where the subscripts ST and X denote standard and sample, respectively. $\boldsymbol{\Phi}$ is the quantum yield. $\boldsymbol{I}$ represents the measured integrated fluorescence intensity. $\boldsymbol{A}$ is the absorbance at the excitation wavelength, and $\boldsymbol{\eta}$ is the refractive index of the solvent. The refractive index for the standard and sample is 1.33. The GQDs and QS were excited at $360 \mathrm{~nm}$ and $310 \mathrm{~nm}$, respectively and the fluorescence intensities were recorded at the emission range of 380-650 $\mathrm{nm}$.

\section{EDC/NHS coupling}

An EDC/NHS conjugation method was used to bind GQDs onto the surface of nanoparticles. For this process, 4.0 $\mathrm{mL}$ of the nanoparticle solution (BIN or BNIN) was first mixed with $4 \mathrm{~mL}$ of graphene quantum dot solution and the resulting solution was mixed with $0.50 \mathrm{~mL}$ of $400 \mu \mathrm{M}$ EDC solution and $0.50 \mathrm{~mL}$ of $400 \mu \mathrm{M}$ NHS solution and stirred at $200 \mathrm{rpm}$ at $25{ }^{\circ} \mathrm{C}$ for $12 \mathrm{~h}$. For removal of the excess EDC/NHS and unreacted graphene quantum dots, the solution was centrifuged for $2 \mathrm{~h}$ at $15000 \mathrm{rpm}$ and the precipitate (activated nanoparticles) was dissolved in $4 \mathrm{~mL}$ ultra-pure water. The cleaning process was carried out by subsequent precipitation and dispersion. The fluorescent composite nanoparticles (GBIN and GBNIN) were dispersed in Milli-Q water after cleaning and stored at $4{ }^{\circ} \mathrm{C}$ in an aluminum foil wrapped glass sample container.

\section{BPA detection procedure and real sample assay}

The detection of BPA was performed in aqueous solution or on nitrocellulose paper. The fluorescence measurements in both approaches were recorded at wavelengths from 400 to $500 \mathrm{~nm}$ at $5 \mathrm{~nm}$ intervals at $25^{\circ} \mathrm{C}$ by excitation at $350 \mathrm{~nm}$. The emission wavelength was determined to be $440 \mathrm{~nm}$. In aqueous solution assay, 150.0 $\mu \mathrm{L}$ nanocomposite solution $\left(1.0 \mathrm{mg} \cdot \mathrm{mL}^{-1}\right.$, in Milli-Q water) and $150 \mu \mathrm{L}$ BPA solutions (different concentrations from $1000 \mathrm{ppb}$ to $50 \mathrm{ppb}$ ) were added into a 96-well fluorescence microplate and mixed $30 \mathrm{~s}$ before the first reading. Photoluminescence intensities of the corresponding suspension were scanned every 30 min for three hours. After investigating the performance of BPA nanosensor in aqueous solution, a paper-based sensing system was also developed to detect BPA via nanocomposites. A standard paper puncher was used to cut nitrocellulose (NC) membrane into circular pieces of $\sim 6 \mathrm{~mm}$ diameter. After placing into a 96-well fluorescence microplate, 2.5 $\mu \mathrm{L}$ of NPs-GQDs solution $\left(1.0 \mathrm{mg} \cdot \mathrm{mL}^{-1}\right.$, in Milli-Q water) were manually pipetted onto each disk. Afterwards, the NC disk was dried for $10 \mathrm{~min}$ at $40{ }^{\circ} \mathrm{C}$ and photoluminescence intensities of the test zones were recorded as an initial value (peaking at $440 \mathrm{~nm}$ ). Then, $2.5 \mu \mathrm{L}$ BPA solutions in tap water or seawater (various concentrations from $0.97 \mathrm{ppb}$ to $1000 \mathrm{ppb}$ ) were dropped onto the test disk. After drying for $5 \mathrm{~min}$ at $40{ }^{\circ} \mathrm{C}$, the intensities of disks were recorded as a final value. Tap water or seawater was used instead of BPA solution to obtain a reference signal (control experiment). Moreover, the disks were treated with only tap water or seawater to eliminate the background signal and the background signal as a blank was subtracted from the measurements in the control and test experiments to eliminate the background signal of nitrocellulose. Preparation and application of the paperbased sensing system was illustrated in Fig. S3. 
Seawater samples from the Icaria shorts, Barcelona (Spain) were filtered by Whatman no. 1 filter paper to remove insoluble impurities. The typical seawater composition was given in Table S2 to take into account the substances that may interfere. The seawater and tap water were also spiked with aminophenol, phenol, hydroxyquinone and naphthol standard solutions instead of BPA to test the specificity and selectivity of the sensor.

The paper-based sensing system was applied to detect BPA in the real samples. The aforementioned fluorescence intensity measurements were performed by using a microplate reader at the excitation and emission wavelengths of $360 \mathrm{~nm}$ and $440 \mathrm{~nm}$, respectively. The real sample assay was carried out as follow: (1) reading the fluorescent intensity of NC disk as blank, (2) pipetting $2.5 \mu \mathrm{L}$ of NPs-GQDs solution (1.0 mg $\cdot \mathrm{mL}-1$, in Milli-Q water) onto the test disk and recording the photoluminescence intensities of the test zones as an initial value after drying for 5 min at $40{ }^{\circ} \mathrm{C}$, (3) pipetting $2.5 \mu \mathrm{L}$ of BPA solution $\left(0.50 \mathrm{mg} \cdot \mathrm{mL}^{-1}\right.$, in tap water or seawater) or analog solutions (10 $\mathrm{mg} \cdot \mathrm{L}^{-1}$, in tap water or seawater) onto test disk and recording the photoluminescence intensities of the test zones as an final value after drying for $5 \mathrm{~min}$ at $40^{\circ} \mathrm{C}$, (4) calculation of the relative response by the below formula.

$$
\text { Relative response }\left(I_{R}\right)=\left[\frac{\left[I / I_{o}\right]_{t}-\left[I / I_{o}\right]_{c}}{\left[I / I_{o}\right]_{c}}\right]
$$

where, $I$ and $I_{o}$ are initial and equilibrium photoluminescence intensities of the sample (maximum emission intensity at $440 \mathrm{~nm}$ ), $c$ is the control experiment and $t$ is the experiment with different analyte concentrations. The results were averaged and the standard deviations were calculated. Means and standard deviations for each test condition were calculated. The results with standard deviations were reported as an average of ten measurements.

\section{Results and Discussion}

\section{Characterization of nanoparticles}

X-ray photoelectron spectroscopy (XPS) and Fourier transformed infrared spectroscopy (FT-IR) were used to study the surface chemical composition and structural analysis of nanoparticles after surface modification of NPs with GQDs (Fig. S4). Smoothed and baseline-corrected FTIR spectra of GBIN, BIN, and GQDs are given in Fig. S4a. According to the FTIR spectra of GBIN and BIN, N-H stretching bands at around $3440 \mathrm{~cm}^{-1}$, the aliphatic C$\mathrm{H}$ bending at $2950 \mathrm{~cm}^{-1}, \mathrm{C}=\mathrm{O}$ stretching at around $1700 \mathrm{~cm}^{-1}$, and $\mathrm{C}-\mathrm{O}$ stretching bands at around $1145 \mathrm{~cm}^{-1}$ originated from EDMA and AAm. The C-N stretching bands at $1320 \mathrm{~cm}^{-1}$ and $1540 \mathrm{~cm}^{-1}$ from VIM indicated the inclusion of this functional monomer into the structure of BIN and GBIN. The structure of GQDs was identified by the characteristic bands in FTIR spectra its (Fig. S5). The increased stretching vibration intensity of $\mathrm{N}-\mathrm{H}$ and $\mathrm{O}-\mathrm{H}$ (wide peak around $3360 \mathrm{~cm}^{-1}$ ) in GBIN spectrum indicated that the surface density of amine and carboxyl groups increased with the immobilization of GQDs by EDC/NHS coupling. Moreover, the structural analysis after the inclusion of the functional monomer into the BIN and BNIN structure was carried out and compared with FTIR analysis provided in Electronic Supplementary Material (Fig. S6). XPS spectra of GQDs, BIN and GBIN were recorded for the advanced chemical structure analysis (Fig. S4b). In XPS spectra, C 1s, O 1s and N 1s signals were investigated for the identification of structure and structural change of nanoparticles. As seen in the XPS spectra, C 1s, N 1s, and O 1s signals were obtained at 288, 533 and $401 \mathrm{eV}$ for GQDs, respectively. C 1s and O 1s signals at $287 \mathrm{eV}$ and $534 \mathrm{eV}$ originate from EDMA and AAm; $\mathrm{N}$ 1s signals at $402 \mathrm{eV}$ originate from VIM and AAm for BIN. In the XPS spectra of GBIN, C 1s, O 1s, and N 1s signals were observed at the same energy values as for BIN, however, the signal intensities were different. In order to understand the evolution of changes clearly, the percent atomic concentrations were calculated from the peak areas of the XPS spectra (Table S3 in the 
Electronic Supplementary Material). The oxygen and nitrogen amounts increased when the amount of carbon was reduced in the structure of GBIN after immobilization of GQDs since GQDs were rich in oxygen and nitrogen. Consequently, these results demonstrate that the nanoparticles were successfully synthesized with the inclusion of functional monomer into the structure and GQDs were immobilized on the nanoparticle surface.

Zeta-sizer analysis, TEM and STEM images were used for the characterization of nanoparticles by surface morphology, shape and size distribution. STEM and TEM images are shown in Fig. 2. Moreover, Zeta-sizer measurements and the size distribution histogram of nanoparticles are provided in Supplementary Material (Fig. S7 and S8). The average size of GQDs was determined to be around $3 \mathrm{~nm}$ according to the size distribution histogram (Fig. S7). As seen in Fig. 2a, the GQDs were spherical with a narrow size distribution and GQDs have crystal lattices with $0.28 \mathrm{~nm}$ size because of $\mathrm{sp}^{2}$ clusters in GQDs. The sizes of BIN and BNIN nanoparticles range from 10 to $50 \mathrm{~nm}$ and the mean sizes are around $25 \mathrm{~nm}$ according to the size distribution histogram and TEM images (Fig. S8). Aqueous solutions of the nanoparticles were used in Zeta-sizer analysis and the nanoparticles swelled in aqueous solution. Therefore, the sizes of the nanoparticles by TEM were found to be smaller than the sizes determined by Zeta-sizer (Fig. S8). Fig. 2b shows that spherical BIN with narrow size distribution, and BNIN with shape and size characteristics similar to BIN (Fig. S8). After surface modification of the BIN and BNIN with GQDs, the nanoparticles maintained their spherical shape and their sizes increased due to coating with GQDs. As seen in Fig. 2c, the nanocomposites (GBIN) formed after the nanoparticles were coated with GQDs have a core-shell structure in which the NP core and GQD shell on the particle can be clearly observed. The mean diameter of GBINs was around $30 \mathrm{~nm}$ with the thickness of GQDs shell around $5 \mathrm{~nm}$. Moreover, the nanocomposites display highly spherical shape and the zeta-sizer measurements of the composite materials confirm these results. The results show that nanoparticles with spherical shape and narrow size distribution were successfully synthesized and GQDs were covalently immobilized onto the nanoparticles. SEM images of the cellulose membrane clearly confirmed the immobilization of GMINs on the cellulose membrane (Fig. 2d) after drying. 


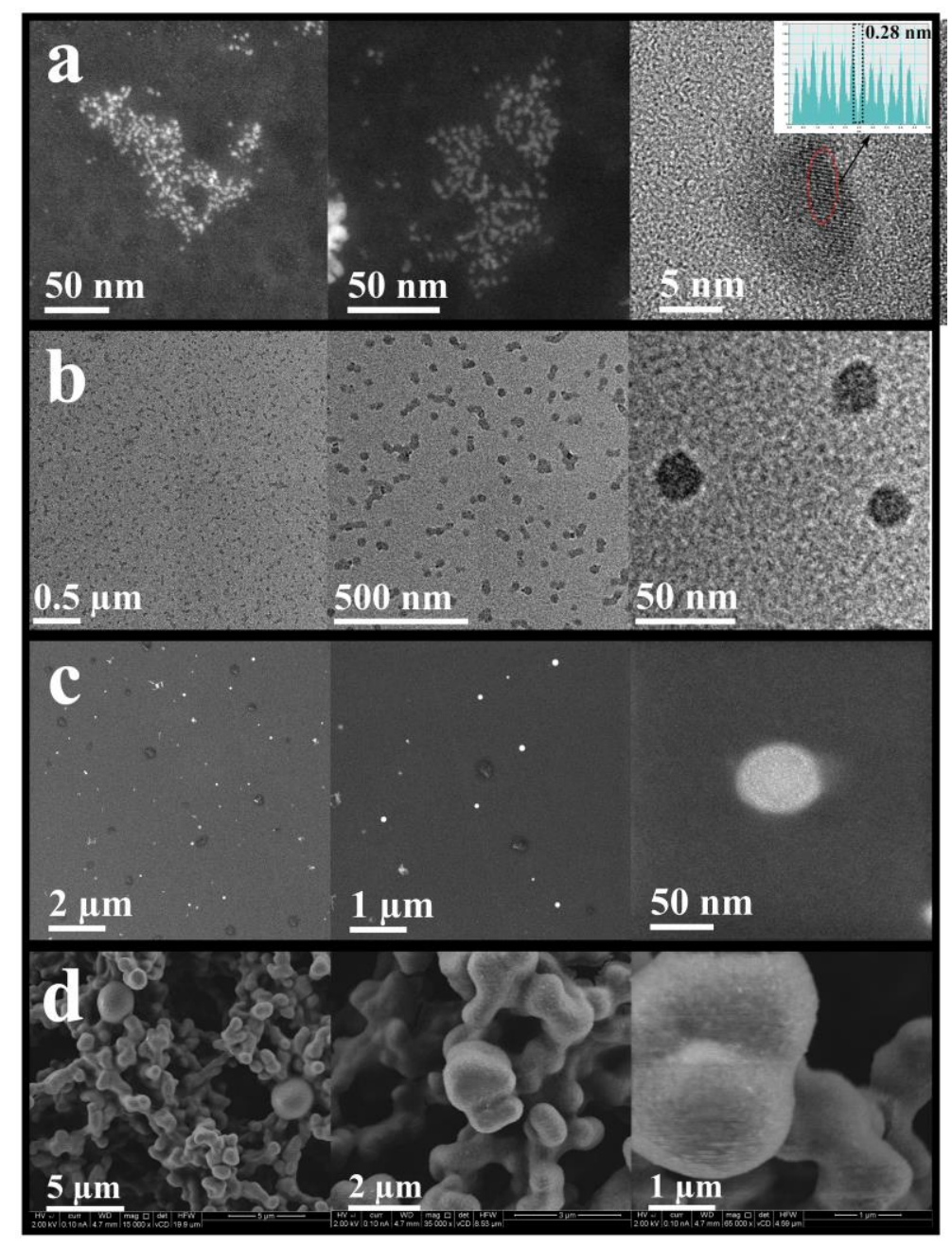

Fig. 2. TEM images of GQDs (a) and BIN (b); STEM images of GBIN (c), and SEM images of GMIN immobilized nitrocellulose membrane $(\mathrm{d})$.

Spectrofluorometric analysis was carried out to investigate the optical properties of nanoparticles before and after the EDC/NHS coupling reaction between nanoparticles and GQDs. UV-VIS absorption and photoluminescence spectra of the GQDs are provided in in the Electronic Supplementary Material (Fig. S9). Two obvious peaks at 280 and $340 \mathrm{~nm}$ are observed in the absorption spectrum and one peak at $440 \mathrm{~nm}$ appears in the emission spectrum (Fig. S9a). The peaks in the absorption spectrum represent two transitions; $\mathrm{p}-\mathrm{p}^{*}$ and $\mathrm{sp}^{2}$ clusters in the structure. The color of the GQDs was blue under a UV lamp (360 nm) (Fig. S9b). The fluorescence quantum yield of the GQDs was calculated to further explore the fluorescence features of the GQDs. The quantum yield of GQDs was calculated using quinine sulfate as a reference to be $88 \%$ with absorbance and fluorescence intensity at excitation and emission wavelengths of $360 \mathrm{~nm}$ and $440 \mathrm{~nm}$, respectively. The emission spectra of nanoparticles were taken at $350 \mathrm{~nm}$ excitation wavelength and the photographs of aqueous solutions of nanoparticles were captured under a $365 \mathrm{~nm}$ UV lamp (Fig. 3). As seen in Fig. 3, BIN and BNIN had no fluorescence properties before coupling, but gained fluorescent properties after the coupling. Moreover, they were visible visually by excitation under a 365 nm UV lamp. The results prove that the GQDs were successfully bound to the surface of BIN and BNIN. 


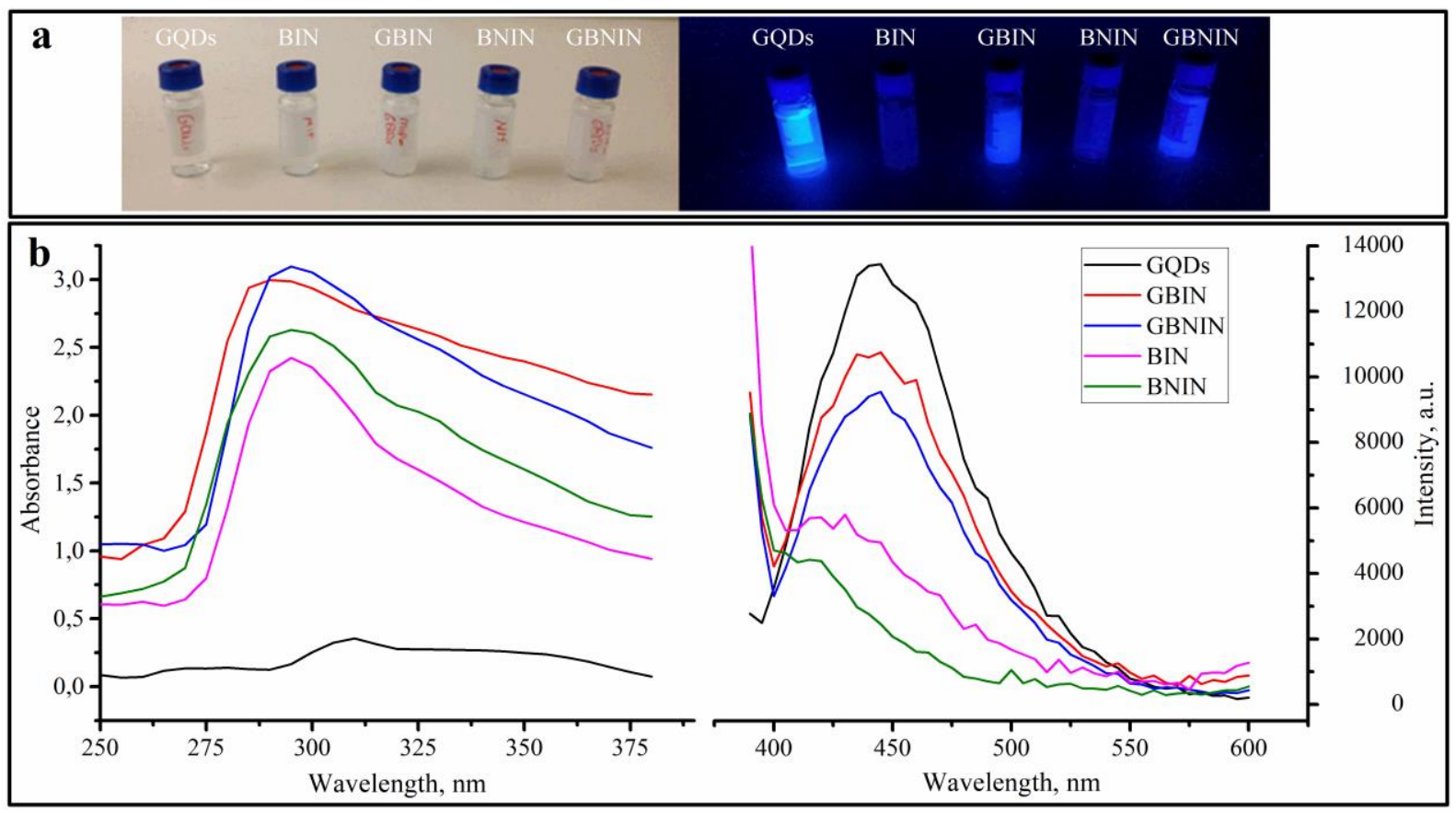

Fig. 3. Optical characterization of nanoparticles: (a) optical photographs under daylight (left) and $365 \mathrm{~nm}$ UV lamp (right); (b) the absorption (left) spectra and the fluorescence (right) spectra at excitation wavelengths of $360 \mathrm{~nm}$.

\section{Detection strategy for BPA}

The photoluminescent sensor was applied to detect BPA first in Milli-Q water. BPA level was determined according to the emission spectrum of NP@GQDs composite with excitation at $350 \mathrm{~nm}$ originally after being incubated with BPA. The emission intensities of GMIN at $440 \mathrm{~nm}$ gradually decreased after treatment with BPA. Therefore, a method based on changes in fluorescence emission was applied to detect the amount of BPA in solution and the response of the nanosensor was calculated as a relative one. Firstly, the response time of the nanosensor was tested by photoluminescence (PL) measurements taken at different times up to $120 \mathrm{~min}$ (Fig. S10a in the Electronic Supplementary Material). The BPA sensing mechanism based on electron-transfer-induced fluorescence quenching and the quenching of the PL signal was materialized by binding of BPA in specific cavities on the surface of BIN. Moreover, the quenching mechanism was also investigated by the Stern-Volmer plots in the Electronic Supplementary Material (Figure S10). The Stern-Volmer plots indicate that there are two populations of fluorophore on the surface of nanoparticles. The response of the nanosensor to BPA increased with increasing incubation time and reached a plateau after $30 \mathrm{~min}$. Therefore, the optimal incubation time was determined to be $30 \mathrm{~min}$ and the GMIN was incubated with BPA for $30 \mathrm{~min}$ in further experiments. The responses of the sensor obtained following the interaction with different BPA concentrations are presented in Fig. S11a; the relative response increases proportionally with increasing BPA concentration in the $50-1000 \mu \mathrm{g} \cdot \mathrm{L}^{-1} \mathrm{range}$ in $\mathrm{Milli}$ Q water. When the relation between the response of nanosensor and BPA concentrations was investigated to find the best fitting curve, the logarithmic curve was defined as the calibration plot (Fig. S11b). The response of the nanosensor is higher at the lower concentrations than at the higher concentrations because the sensor response decreases at higher BPA concentrations due to steric hindrance and cavities filled to saturation. The LOD value of GBIN nanosensor in the aqueous solution were determined as $43.9 \pm 0.8 \mu \mathrm{g} \cdot \mathrm{L}^{-1}$ according to the equation in Figure $11 \mathrm{~b}$. 
After investigating the performance of the BPA nanosensor in aqueous solution, a paper-based sensing system (GMIN@NC) for BPA detection was also developed by the application of NP@GQD nanocomposite materials to a nitrocellulose surface. Fig. 4 and 5 show that the response of GBIN@NC and GBNIN@NC for different concentrations of BPA range from 0.97 to $1000 \mu \mathrm{g} \cdot \mathrm{L}^{-1}$ in seawater and tap water. The relationship between BPA concentration and the response of the nanosensor in tap water and seawater is shown by the calibration plots. The paper-based sensing system responded to low sample volume $(2.5 \mu \mathrm{L})$ with a shorter response time compared to the sensing performance in aqueous solutions. As seen in Fig. 4 and 5, the response of the system increased with increasing BPA concentration, thus BPA detection in tap water and seawater was successfully performed. When the relationship between different BPA concentrations and the response of system is investigated, the response of the nanosensor increases as the logarithmic function of the concentration. The results obtained from the paperbased sensor were in agreement with the response of the sensor in the aqueous phase. However, some matrixes such as blood, serum, cells, marine water, wastewaters etc. always display strong background UV absorption and fluorescence and this causes signal attenuation in colorimetric and fluorometric sensors and therefore, the response of the sensor in seawater is lower than tap water. The LOD values of GBIN@NC and GBNIN@NC in tap water and seawater samples were calculated by using the equation (Table 1). As seen clearly in Table 1, even in the presence of a number of interferences such as those contained in seawater, GBIN composite materials have a very low LOD value $\left(4.2 \pm 0.5 \mu \mathrm{g} \cdot \mathrm{L}^{-1}\right)$ as a result of application to the nitrocellulose surface. The GBIN nanosensor has at high response capacity to BPA due to the use of BPA-specific recognition sites and the response of GBNIN is likely caused by non-specific interactions such as $\pi$ stacking and, van der Waals interaction. 

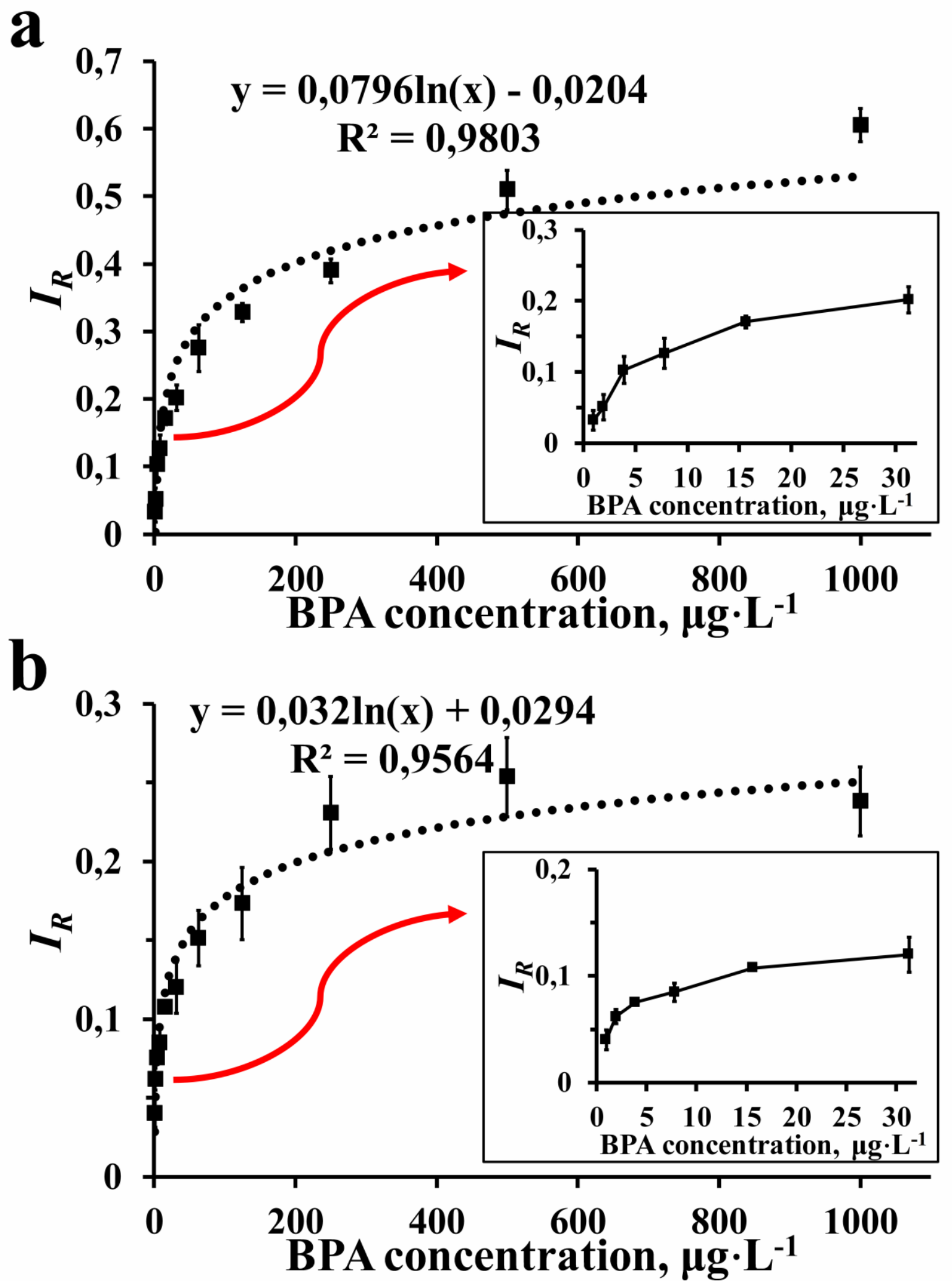

Fig. 4. Response of GBIN (a, b) and $\operatorname{GBNIN~(c,d)~paper-based~nanosensor~systems~for~various~concentrations~of~}$ BPA in tap water. Fluorescence intensity was measured using a microplate reader at excitation and emission wavelengths of $360 \mathrm{~nm}$ and $440 \mathrm{~nm}$, respectively. Error bars correspond to the standard deviation of the mean for $\mathrm{n}=10$. 


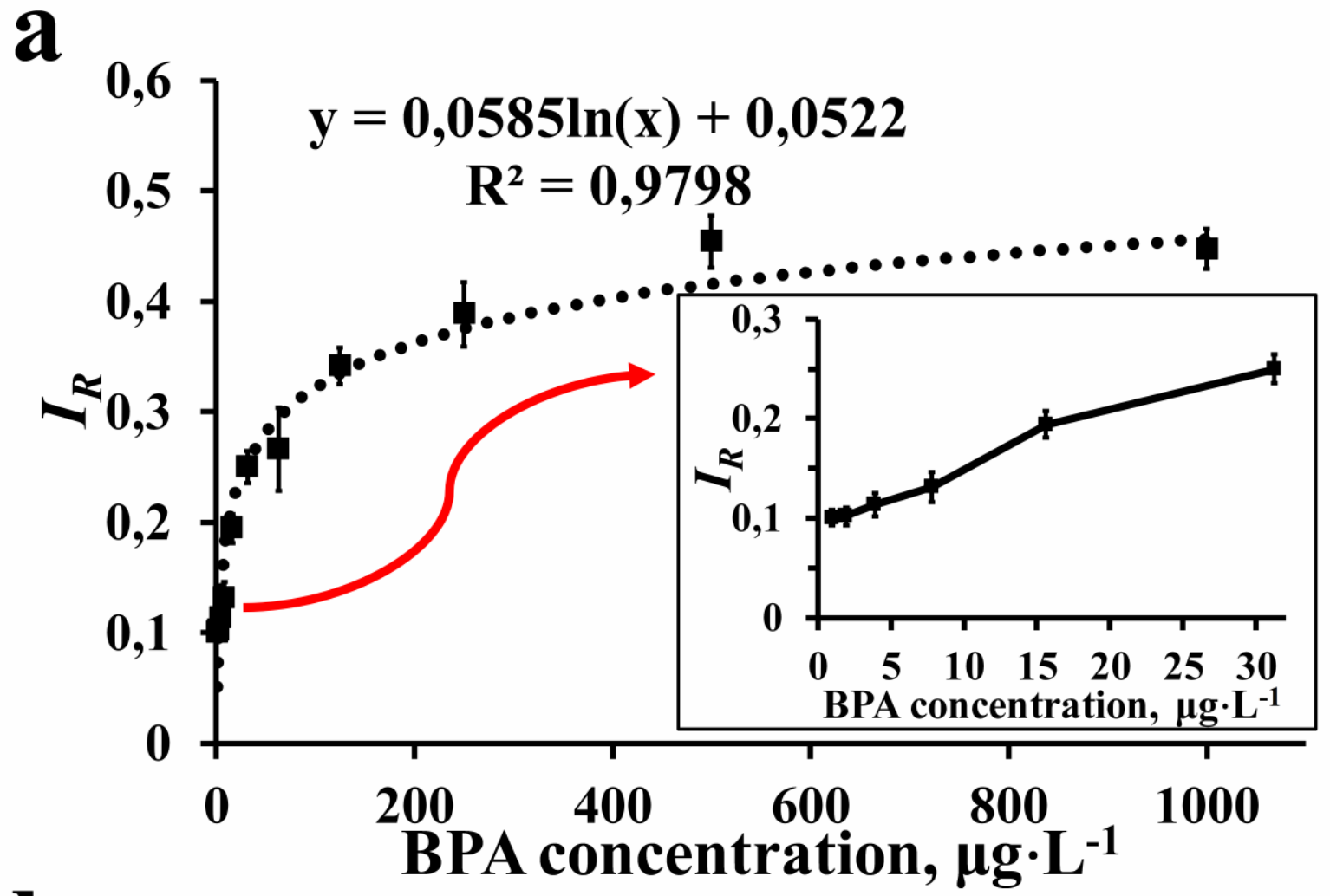

b

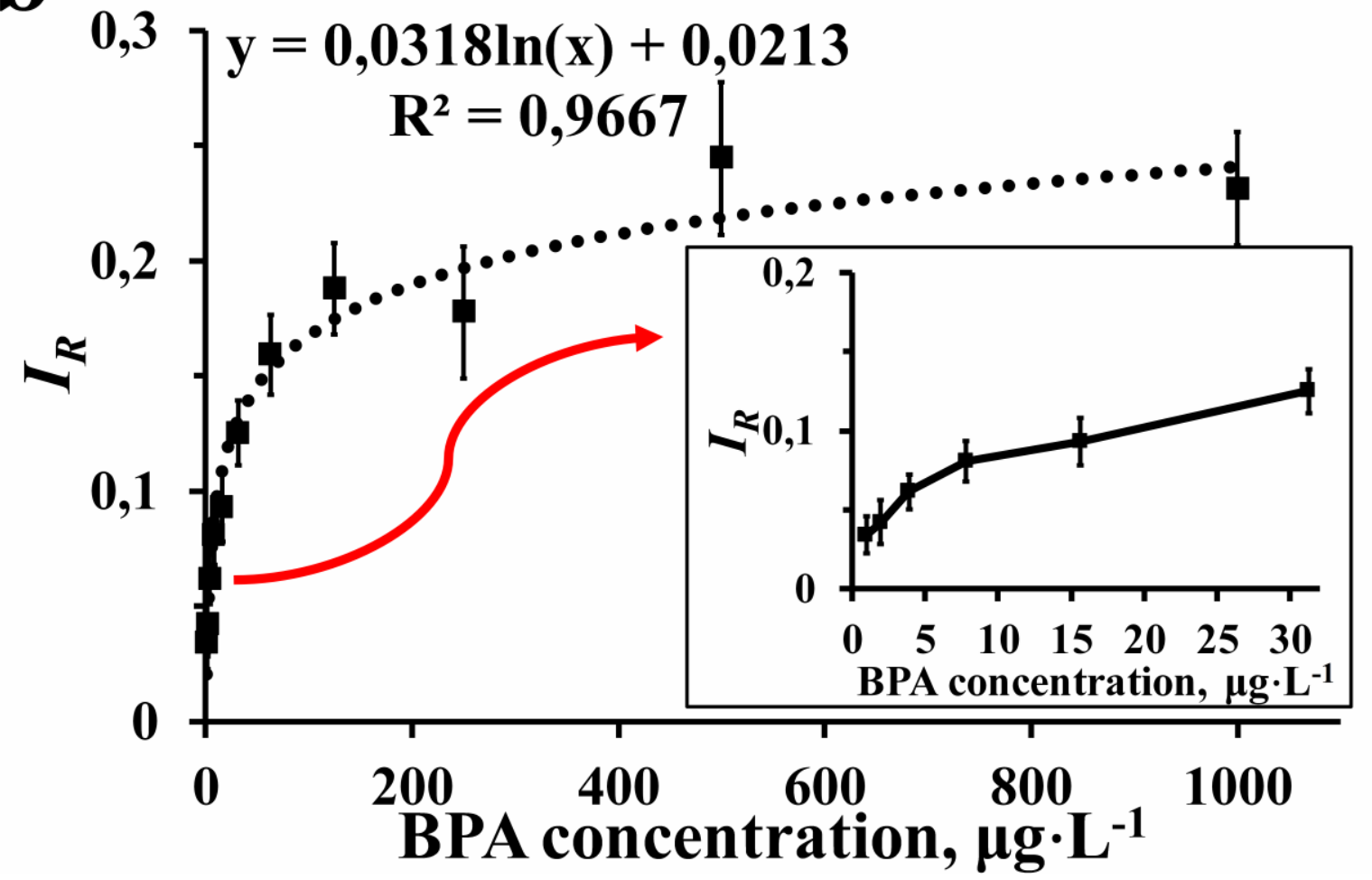

Fig. 5. Response of GBIN (a, b) and GBNIN (c, d) paper-based nanosensor systems for various concentrations of BPA in seawater. Fluorescence intensity was measured using a microplate reader at excitation and emission wavelengths of $360 \mathrm{~nm}$ and $440 \mathrm{~nm}$, respectively. Error bars correspond to the standard deviation of the mean for $\mathrm{n}=10$. 
Table 1. LOD values of GBIN@NC and GBNIN@NC in different media.

\begin{tabular}{|c|c|c|}
\hline & LOD, $\boldsymbol{\mu g} \cdot \mathbf{L}^{-\mathbf{1}}$ & Media \\
\hline \multirow{2}{*}{ GBIN } & $1.8 \pm 0.2 \mu \mathrm{g} \cdot \mathrm{L}^{-1}$ & Tap water \\
\cline { 2 - 3 } & $4.2 \pm 0.5$ & Seawater \\
\hline \multirow{2}{*}{ GBNIN } & $171.1 \pm 1.9$ & Tap water \\
\cline { 2 - 3 } & $3.7 \times 10^{3} \pm 25.5$ & Seawater \\
\hline
\end{tabular}

The selectivity and specificity of the sensor system for BPA detection in tap water and seawater were investigated using BPA as well as its structural analogs such as aminophenol, phenol, hydroquinone and naphthol. The structures of the analogs are provided in Supplementary material (Fig. S12). Fig. 6 shows the response of GMIN@NC and GBNIN@NC to BPA and structural analogues in tap water and seawater. As seen in Fig. 6, GBIN nanosensors have a negligible response to structural analogs but significant response to BPA, whereas GBNIN nanosensors have a similar response to BPA and structural analogs. The selectivity and relative selectivity coefficients for GBIN@NC and GBNIN@NC were calculated using the relative responses (Table S4 in the Electronic Supplementary Material). In Table S4, the selectivity coefficients of GBIN@NC indicating specificity to BPA instead of structural analogs are greater than 1 proving the selectivity of the system. The relative selectivity coefficients expressing BPA recognition capacity of GBIN relative to GBNIN proves that the imprinting efficiency and factor are high. Consequently, GBIN@NC provides satisfactory results in BPA assay in terms of sensitivity and selectivity due to the GBIN cavities having the ability to preferentially recognize BPA. 


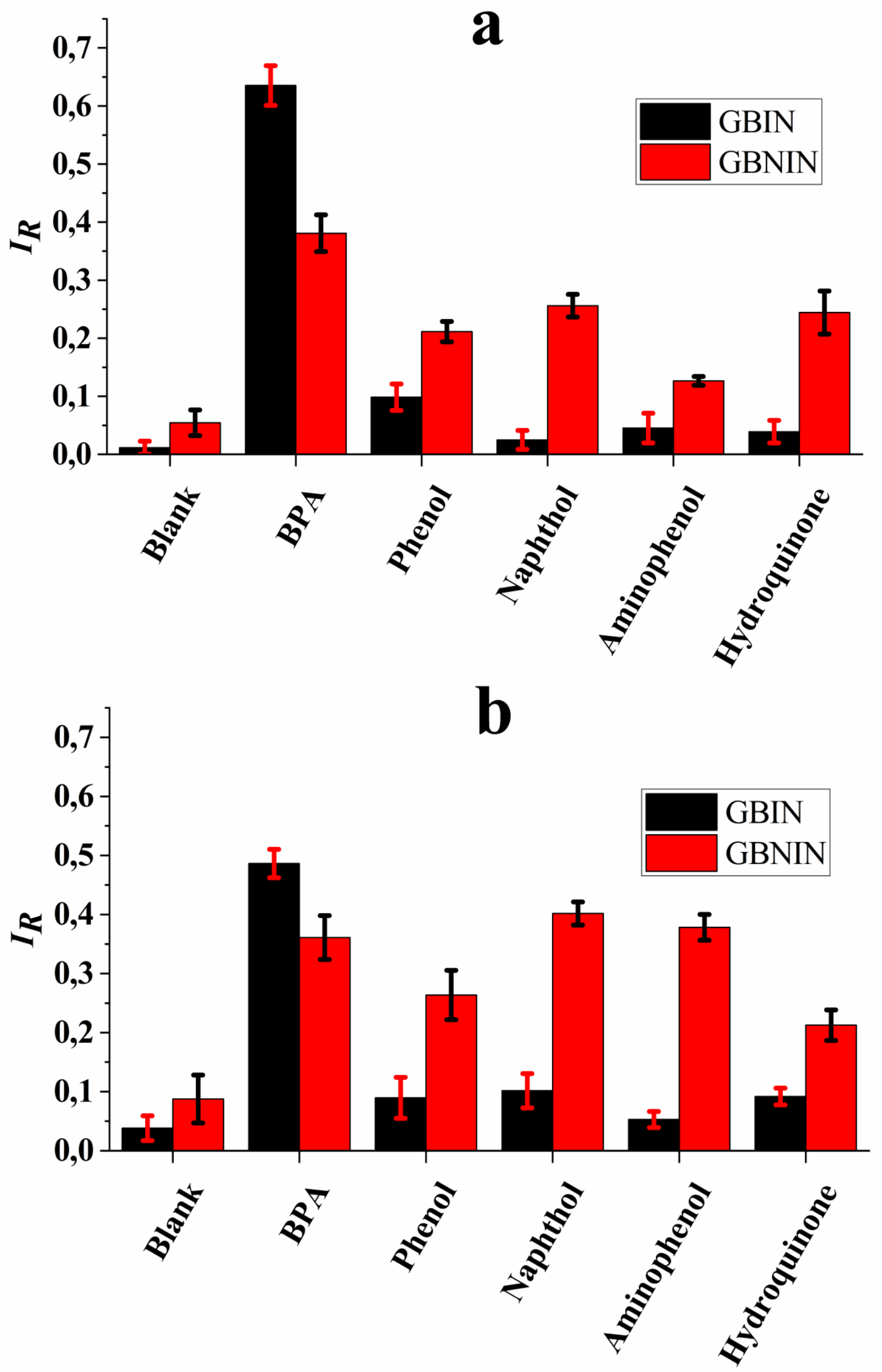


Fig. 6. Comparison of nanosensors: the responses of GBIN@NC and GBNIN@NC in tap water (A) and seawater (B). Fluorescence intensity was measured using a microplate reader at excitation and emission wavelengths of 360 $\mathrm{nm}$ and $440 \mathrm{~nm}$, respectively. Error bars correspond to the standard deviation of the mean for $\mathrm{n}=10$.

The methods for BPA detection in literature are summarized in Table S5 in the Electronic Supplementary Material. The molecular imprinting technique has been used in some studies to provide selective sensors to be used in BPA determination. As seen in Table S5, the LOD obtained from this study is lower than the values reported for many other studies. Moreover, the GBIN@NC system is not only easy to use, but also has the advantage of working with very low sample volumes $(2.5 \mu \mathrm{L})$ for determination of BPA in various sources.

\section{Conclusion}

In this study, a fluorescent sensor for the sensitive and selective detection of BPA was developed. NPs were prepared by the mini-emulsion polymerization method and GQDs were synthesized by hydrothermal pyrolysis. GQDs were attached to the NPs by EDC/NHS coupling. Fluorescent nanosensors were characterized by Zetasizer, UV-VIS and photoluminescence spectra, TEM and FTIR. The nanosensors responded to BPA as an enhancement of the PL intensity. The detection limit and selectivity of the fluorescent nanosensor were found to be comparable to those of other techniques.

In summary, we developed a paper-based sensing system that successfully achieves, high selectivity of MIPs, and imparts the fluorescence properties of GQDs to develop a selective, sensitive, rapid and inexpensive sensing strategy for BPA monitoring. In addition, the sensing platform that can be exploited using small volumes of the nanocomposite and the sample. This test stripe can be easily produced because of its easy preparation, low cost, lack of toxicity, and environmentally friendliness. Moreover, the sensing system can be easily extended to monitor not only pollutants but also biomolecules by simply choosing different functional monomers for the molecular imprinting techniques.

\section{Acknowledgments}

Recep Üzek thanks to TUBITAK for the given scholarship. We acknowledge support from MINECO, Spain for MAT2017-87202-P and Graphene Flagship Core Project 2 (Ref: 785219). ICN2 is supported by the Severo Ochoa program from Spanish MINECO (Grant No. SEV-2017-0706). This work is also funded by the CERCA Programme / Generalitat de Catalunya.

\section{Compliance with ethical standards}

The author(s) declare that they have no competing interests.

\section{References}

1. Vandenberg LN, Hauser R, Marcus M, Olea N, Welshons WV (2007) Human exposure to bisphenol A (BPA). Reprod Toxicol 24 (2):139-177

2. Brede C, Fjeldal P, Skjevrak I, Herikstad H (2003) Increased migration levels of bisphenol A from polycarbonate baby bottles after dishwashing, boiling and brushing. Food Addit Contam 20 (7):684-689

3. Maia J, Cruz J, Sendón R, Bustos J, Sanchez J, Paseiro P (2009) Effect of detergents in the release of bisphenol A from polycarbonate baby bottles. Food Res Int 42 (10):1410-1414

4. Burridge E (2003) Bisphenol A: product profile. Eur Chem News 17:14-20

5. Staples CA, Dome PB, Klecka GM, Oblock ST, Harris LR (1998) A review of the environmental fate, effects, and exposures of bisphenol A. Chemosphere 36 (10):2149-2173 
6. Wozniak AL, Bulayeva NN, Watson CS (2005) Xenoestrogens at picomolar to nanomolar concentrations trigger membrane estrogen receptor- $\alpha$-mediated $\mathrm{Ca} 2+$ fluxes and prolactin release in $\mathrm{GH} 3 / \mathrm{B} 6$ pituitary tumor cells. Environ Health Perspect:431-439

7. Vandenberg LN, Maffini MV, Sonnenschein C, Rubin BS, Soto AM (2009) Bisphenol-A and the great divide: a review of controversies in the field of endocrine disruption. Endocr Rev 30 (1):75-95

8. Wolstenholme JT, Rissman EF, Connelly JJ (2011) The role of Bisphenol A in shaping the brain, epigenome and behavior. Horm Behav 59 (3):296-305

9. Tharp AP, Maffini MV, Hunt PA, VandeVoort CA, Sonnenschein C, Soto AM (2012) Bisphenol A alters the development of the rhesus monkey mammary gland. Proc Natl Acad Sci 109 (21):8190-8195

10. Watabe Y, Kondo T, Morita M, Tanaka N, Haginaka J, Hosoya K (2004) Determination of bisphenol A in environmental water at ultra-low level by high-performance liquid chromatography with an effective on-line pretreatment device. J Chromatogr A 1032 (1):45-49

11. Kim A, Li C-R, Jin C-F, Lee KW, Lee S-H, Shon K-J, Park NG, Kim D-K, Kang S-W, Shim Y-B (2007) A sensitive and reliable quantification method for bisphenol A based on modified competitive ELISA method. Chemosphere 68 (7):1204-1209

12. Xue F, Wu J, Chu H, Mei Z, Ye Y, Liu J, Zhang R, Peng C, Zheng L, Chen W (2013) Electrochemical aptasensor for the determination of bisphenol A in drinking water. Microchim Acta 180 (1-2):109-115

13. Ren X, Cheshari EC, Qi J, Li X (2018) Silver microspheres coated with a molecularly imprinted polymer as a SERS substrate for sensitive detection of bisphenol A. Microchim Acta 185 (4):242

14. Ensafi AA, Amini M, Rezaei B (2018) Molecularly imprinted electrochemical aptasensor for the attomolar detection of bisphenol A. Microchim Acta 185:265

15. Goulart LA, Gonçalves R, Correa AA, Pereira EC, Mascaro LH (2018) Synergic effect of silver nanoparticles and carbon nanotubes on the simultaneous voltammetric determination of hydroquinone, catechol, bisphenol A and phenol. Microchim Acta 185 (1):12

16. Kawaguchi M, Ito R, Endo N, Okanouchi N, Sakui N, Saito K, Nakazawa H (2006) Liquid phase microextraction with in situ derivatization for measurement of bisphenol $\mathrm{A}$ in river water sample by gas chromatography-mass spectrometry. J Chromatogr A 1110 (1):1-5

17. Fenlon KA, Johnson AC, Tyler CR, Hill EM (2010) Gas-liquid chromatography-tandem mass spectrometry methodology for the quantitation of estrogenic contaminants in bile of fish exposed to wastewater treatment works effluents and from wild populations. J Chromatogr A 1217 (1):112-118

18. Li X, Franke AA (2015) Improvement of bisphenol A quantitation from urine by LCMS. Anal BioanalChem 407 (13):3869-3874

19. Mei Q, Zhang K, Guan G, Liu B, Wang S, Zhang Z (2010) Highly efficient photoluminescent graphene oxide with tunable surface properties. Chem Commun 46 (39):7319-7321

20. Zheng XT, Than A, Ananthanaraya A, Kim D-H, Chen P (2013) Graphene quantum dots as universal fluorophores and their use in revealing regulated trafficking of insulin receptors in adipocytes. ACS nano 7 (7):6278-6286

21. Pan D, Zhang J, Li Z, Wu M (2010) Hydrothermal route for cutting graphene sheets into blue-luminescent graphene quantum dots. Adv Mater 22 (6):734-738

22. Baker SN, Baker GA (2010) Luminescent carbon nanodots: emergent nanolights. Angewandte Chemie International Edition 49 (38):6726-6744

23. Shen J, Zhu Y, Chen C, Yang X, Li C (2011) Facile preparation and upconversion luminescence of graphene quantum dots. Chem Commun 47 (9):2580-2582

24. Sun H, Wu L, Wei W, Qu XJMT (2013) Recent advances in graphene quantum dots for sensing. 16 (11):433442

25. Li H, He X, Kang Z, Huang H, Liu Y, Liu J, Lian S, Tsang CHA, Yang X, Lee ST (2010) Water-Soluble Fluorescent Carbon Quantum Dots and Photocatalyst Design. Angewandte Chemie International Edition 49 (26):4430-4434

26. Qu D, Zheng M, Du P, Zhou Y, Zhang L, Li D, Tan H, Zhao Z, Xie Z, Sun Z (2013) Highly luminescent S, N co-doped graphene quantum dots with broad visible absorption bands for visible light photocatalysts. Nanoscale 5 (24):12272-12277

27. Sun X, Liu Z, Welsher K, Robinson JT, Goodwin A, Zaric S, Dai H (2008) Nano-graphene oxide for cellular imaging and drug delivery. Nano research 1 (3):203-212

28. Lin Y, Chapman R, Stevens MM (2015) Integrative Self-Assembly of Graphene Quantum Dots and Biopolymers into a Versatile Biosensing Toolkit. Adv Funct Mater 25. (21):3183-3192

29. Nigam P, Waghmode S, Louis M, Wangnoo S, Chavan P, Sarkar D (2014) Graphene quantum dots conjugated albumin nanoparticles for targeted drug delivery and imaging of pancreatic cancer. Journal of Materials Chemistry B 2 (21):3190-3195 
30. Su Z, Shen H, Wang H, Wang J, Li J, Nienhaus GU, Shang L, Wei G (2015) Motif-Designed Peptide Nanofibers Decorated with Graphene Quantum Dots for Simultaneous Targeting and Imaging of Tumor Cells. Adv Funct Mater 25 (34):5472-5478

31. Bereli N, Andaç M, Baydemir G, Say R, Galaev IY, Denizli A (2008) Protein recognition via ion-coordinated molecularly imprinted supermacroporous cryogels. J Chromatogr A 1190 (1):18-26

32. Yin J, Yang G, Chen YJJoCA (2005) Rapid and efficient chiral separation of nateglinide and its l-enantiomer on monolithic molecularly imprinted polymers. 1090 (1-2):68-75

33. Birlik E, Ersöz A, Denizli A, Say R (2006) Preconcentration of copper using double-imprinted polymer via solid phase extraction. Analytica chimica acta 565 (2):145-151

34. Uzun L, Uzek R, Şenel S, Say R, Denizli A (2013) Chiral recognition of proteins having L-histidine residues on the surface with lanthanide ion complex incorporated-molecularly imprinted fluorescent nanoparticles. Mater Sci Eng C 33 (6):3432-3439

35. Chen L, Xu S, Li J (2011) Recent advances in molecular imprinting technology: current status, challenges and highlighted applications. Chem Soc Rev 40 (5):2922-2942

\section{Figure Captions}

Fig. 1. BPA sensing mechanism based on the fluorescence quenching by the binding of targets in specific recognition sites.

Fig. 2. TEM images of GQDs (a) and BIN (b); STEM images of GBIN (c), and SEM images of GMIN immobilized nitrocellulose membrane (d).

Fig. 3. Optical characterization of nanoparticles: (a) optical photographs under daylight (left) and $365 \mathrm{~nm}$ UV lamp (right); (b) the absorption (left) spectra and the fluorescence (right) spectra at excitation wavelengths of $360 \mathrm{~nm}$.

Fig. 4. Response of GBIN (a, b) and GBNIN (c, d) paper-based nanosensor systems for various concentrations of BPA in tap water. Fluorescence intensity was measured using a microplate reader at excitation and emission wavelengths of $360 \mathrm{~nm}$ and $440 \mathrm{~nm}$, respectively. Error bars correspond to the standard deviation of the mean for $\mathrm{n}=10$.

Fig. 5. Response of GBIN (a, b) and GBNIN (c, d) paper-based nanosensor systems for various concentrations of BPA in seawater. Fluorescence intensity was measured using a microplate reader at excitation and emission wavelengths of $360 \mathrm{~nm}$ and $440 \mathrm{~nm}$, respectively. Error bars correspond to the standard deviation of the mean for $\mathrm{n}=10$.

Fig. 6. Comparison of nanosensors: the responses of GBIN@NC and GBNIN@NC in tap water (A) and seawater (B). Fluorescence intensity was measured using a microplate reader at excitation and emission wavelengths of 360 $\mathrm{nm}$ and $440 \mathrm{~nm}$, respectively. Error bars correspond to the standard deviation of the mean for $\mathrm{n}=10$.

\section{Table Captions}

Table1. LOD values of GBIN@NC and GBNIN@NC in different media.

\section{Graphical Abstract}

Schematic presentation of graphene quantum dots immobilized on molecularly imprinted nanoparticles placed on nitrocellulose paper for the determination of Bisphenol A in tap water and seawater based on the fluorescence quenching by the binding of targets in specific recognition sites. 